\title{
Characterization of a Kinetically Stable, Highly Ordered, Octameric Form of Lithium tert-Butoxide and its Implications Regarding Aggregate Formation
}

\author{
John F. Allan, Roger Nassar, Elizabeth Specht, Alicia Beatty, Nathalie Calin, \\ and Kenneth W. Henderson,
}

Department of Chemistry and Biochemistry, University of Notre Dame, Notre Dame, Indiana 46556, USA

E-mail: khenders@nd.edu

Tables of atomic coordinates and associated energies for the calculated geometries of $(\mathrm{MeOLi})_{6}, \mathbf{I},(\mathrm{MeOLi})_{8}, \mathbf{I I},\left({ }^{t} \mathrm{BuOLi}\right)_{6}, \mathbf{I I I},\left({ }^{\mathrm{t}} \mathrm{BuOLi}\right)_{8}, \mathbf{I V}$. Geometry optimizations and frequency analyses for I and II were performed at the B3LYP/6311G** level. Geometry optimizations and frequency analyses for III and IV were performed at the HF/6-31G level and single point energies recalculated at the B3LYP/6311G ${ }^{* * *}$ level.

All manipulations were carried out using Schlenk techniques or inside an argon-filled glove box. All glassware was flame-dried under vacuum before use. Solvents (hexane and toluene) were dried by passage through copper-based catalyst and molecular sieve columns (Innovative Technology). ${ }^{\mathrm{t}} \mathrm{BuOH}$ was recrystallized from hexane before use. ${ }^{\mathrm{t}} \mathrm{BuOOH}$ was purchased as an anhydrous solution in decane and used as received. ${ }^{\mathrm{t}} \mathrm{BuLi}$ was purchased as either $1.5 \mathrm{M}$ (Acros) or $1.7 \mathrm{M}$ solutions in pentane and were standardized using salicylaldehyde phenylhydrazone.

All variable temperature NMR data was recorded on a Varian INOVA $500 \mathrm{MHz}$ instrument. ${ }^{1} \mathrm{H}$ and ${ }^{13} \mathrm{C}$ spectra were referenced with respect to the residual solvent signal. ${ }^{7} \mathrm{Li}$ spectra were referenced with respect to a $1 \mathrm{M}$ solution on $\mathrm{LiCl}$ in $\mathrm{D}_{2} \mathrm{O}$.

MS analyses were performed on a JEOL GCMate instrument in electron impact mode using a direct injection probe.

Phase identification was done with X-Ray powder diffraction performed on a D8 Brücker diffractometer $(\mathrm{Cu} \mathrm{K} \alpha)$. The patterns were collected in steps of $0.02^{\circ}(2 \theta)$ over the angular range $3-60^{\circ}(2 \theta)$ counting 15 seconds per step. From the calculated crystal structures, determined by single crystal X-ray diffraction, pure powder diffraction patterns were derived using Powder Cell for Windows version 2.4. The theoretical powder patterns were then compared to the actual powder patterns. 
Hexamer, $(\mathrm{MeOLi})_{6}, \mathbf{I}$

Optimization B3LYP/6-311G**: - 736.748140745 h

$\begin{array}{lccc}\mathrm{Li} & -1.167789 & 0.834546 & 1.329502 \\ \mathrm{O} & 0.628521 & 1.054574 & 1.875412 \\ \mathrm{C} & 0.964802 & 1.908218 & 2.938148 \\ \mathrm{Li} & 1.166700 & -0.836052 & -1.328651 \\ \mathrm{O} & -0.629058 & -1.054634 & -1.875247 \\ \mathrm{C} & -0.964548 & -1.903892 & -2.941743 \\ \mathrm{Li} & 1.729105 & 0.834166 & 0.361711 \\ \mathrm{O} & -1.906508 & 1.085177 & -0.381270 \\ \mathrm{O} & 1.906169 & -1.085850 & 0.382093 \\ \mathrm{Li} & -1.729337 & -0.834836 & -0.360941 \\ \mathrm{Li} & 0.559580 & -0.856351 & 1.684224 \\ \mathrm{O} & -1.299120 & -1.083045 & 1.456221 \\ \mathrm{O} & 1.299521 & 1.081976 & -1.455487 \\ \mathrm{Li} & -0.559110 & 0.856410 & -1.682990 \\ \mathrm{C} & -3.013850 & 1.915859 & -0.613167 \\ \mathrm{C} & 3.013268 & -1.916833 & 0.613931 \\ \mathrm{C} & -2.048634 & -1.923412 & 2.294078 \\ \mathrm{C} & 2.049376 & 1.922035 & -2.293315 \\ \mathrm{H} & -0.901553 & -2.969439 & -2.665145 \\ \mathrm{H} & -0.302244 & -1.765861 & -3.813912 \\ \mathrm{H} & -1.991661 & -1.733570 & -3.307858 \\ \mathrm{H} & 2.748119 & -2.986941 & 0.582179 \\ \mathrm{H} & -1.870929 & -2.991328 & 2.083714 \\ \mathrm{H} & -3.135426 & -1.759133 & 2.191966 \\ \mathrm{H} & -1.815325 & -1.769532 & 3.361991 \\ \mathrm{H} & 0.354685 & 1.719258 & 3.838214 \\ \mathrm{H} & 0.827411 & 2.971929 & 2.681717 \\ \mathrm{H} & 2.016569 & 1.794212 & 3.252530 \\ \mathrm{H} & -2.749012 & 2.986035 & -0.581278 \\ \mathrm{H} & -3.814224 & 1.767824 & 0.132447 \\ \mathrm{H} & -3.476687 & 1.735095 & -1.598938 \\ \mathrm{H} & 1.815758 & 1.768523 & -3.361222 \\ \mathrm{H} & 1.872402 & 2.990035 & -2.082718 \\ \mathrm{H} & 3.136089 & 1.757052 & -2.191458 \\ \mathrm{H} & 3.476291 & -1.736120 & 1.599638 \\ \mathrm{H} & 3.813606 & -1.769114 & -0.131793 \\ & & & \\ & & & \\ \mathrm{H} & & & \end{array}$


Octamer, $(\mathrm{MeOLi})_{8}, \mathbf{I I}$

Optimization B3LYP/6-311G**: -982.349195703 h

\begin{tabular}{lrrr}
$\mathrm{C}$ & -1.076405 & -3.350140 & 0.770213 \\
$\mathrm{C}$ & -0.220336 & -2.510332 & -3.390026 \\
$\mathrm{O}$ & -0.440355 & -2.091993 & 0.751576 \\
$\mathrm{Li}$ & 0.620352 & -1.715423 & -0.735989 \\
$\mathrm{O}$ & 0.011901 & -1.445712 & -2.507337 \\
$\mathrm{Li}$ & 0.020753 & -1.215117 & 2.356361 \\
$\mathrm{Li}$ & -1.715549 & -0.620118 & 0.736014 \\
$\mathrm{C}$ & 3.350369 & -1.076044 & -0.770679 \\
$\mathrm{O}$ & -1.445868 & -0.011834 & 2.507422 \\
$\mathrm{Li}$ & -1.214648 & -0.020847 & -2.356373 \\
$\mathrm{C}$ & -2.510526 & 0.220294 & 3.390159 \\
$\mathrm{C}$ & 2.510231 & -0.220781 & 3.390273 \\
$\mathrm{O}$ & 2.092149 & -0.440148 & -0.751716 \\
$\mathrm{O}$ & -2.091916 & 0.440371 & -0.751813 \\
$\mathrm{C}$ & -3.350145 & 1.076230 & -0.771128 \\
$\mathrm{Li}$ & 1.215043 & 0.021029 & -2.356378 \\
$\mathrm{O}$ & 1.445684 & 0.011330 & 2.507409 \\
$\mathrm{Li}$ & 1.715503 & 0.620165 & 0.736173 \\
$\mathrm{Li}$ & -0.020964 & 1.214636 & 2.356542 \\
$\mathrm{O}$ & -0.011475 & 1.445924 & -2.507189 \\
$\mathrm{Li}$ & -0.620090 & 1.715588 & -0.735919 \\
$\mathrm{O}$ & 0.440244 & 2.091956 & 0.752031 \\
$\mathrm{C}$ & 0.220446 & 2.510583 & -3.389979 \\
$\mathrm{C}$ & 1.076036 & 3.350230 & 0.771425 \\
$\mathrm{H}$ & 3.236095 & 0.609928 & 3.400005 \\
$\mathrm{H}$ & 3.080727 & -1.129409 & 3.131493 \\
$\mathrm{H}$ & 2.171107 & -0.347128 & 4.432498 \\
$\mathrm{H}$ & 4.182745 & -0.353789 & -0.758526 \\
$\mathrm{H}$ & 3.496753 & -1.741410 & 0.096282 \\
$\mathrm{H}$ & 3.491625 & -1.697212 & -1.672395 \\
$\mathrm{H}$ & -0.429055 & -2.165565 & -4.417714 \\
$\mathrm{H}$ & 0.644441 & -3.190762 & -3.462972 \\
$\mathrm{H}$ & -1.082187 & -3.128622 & -3.085814 \\
$\mathrm{H}$ & -1.741383 & -3.496418 & -0.097079 \\
$\mathrm{H}$ & -0.354239 & -4.182596 & 0.758357 \\
$\mathrm{H}$ & -1.698016 & -3.491321 & 1.671635 \\
$\mathrm{H}$ & -2.172795 & 0.333199 & 4.434362 \\
$\mathrm{H}$ & -3.072774 & 1.136260 & 3.139295 \\
$\mathrm{H}$ & -3.243447 & -0.604264 & 3.389817 \\
$\mathrm{H}$ & 1.696783 & 3.491374 & 1.673447 \\
$\mathrm{H}$ & 0.353724 & 4.182552 & 0.759072 \\
$\mathrm{H}$ & 1.741739 & 3.496800 & -0.095246 \\
& & & \\
\hline
\end{tabular}




$\begin{array}{lrrr}\mathrm{H} & -0.604575 & 3.242438 & -3.390937 \\ \mathrm{H} & 0.333799 & 2.172133 & -4.434455 \\ \mathrm{H} & 1.137307 & 3.071776 & -3.140721 \\ \mathrm{H} & -3.492346 & 1.694932 & -1.674388 \\ \mathrm{H} & -3.495631 & 1.743935 & 0.094198 \\ \mathrm{H} & -4.182526 & 0.354035 & -0.756123\end{array}$


Hexamer, $\left({ }^{\mathrm{B}} \mathrm{BuOLi}\right)_{6}$, III

HF/6-31G ${ }^{*}$ optimization: -1434.76456837 h

Single Point B3LYP/6-311G**: -1434.76456837 h

\begin{tabular}{lrrr}
$\mathrm{Li}$ & 1.759000 & -0.869207 & 0.001272 \\
$\mathrm{Li}$ & -0.890782 & -0.844711 & -1.532577 \\
$\mathrm{Li}$ & -0.899604 & -0.834581 & 1.541048 \\
$\mathrm{Li}$ & 0.891362 & 0.844668 & -1.532242 \\
$\mathrm{Li}$ & -1.759007 & 0.869209 & 0.000550 \\
$\mathrm{Li}$ & 0.899014 & 0.834637 & 1.541385 \\
$\mathrm{O}$ & 0.978567 & -1.096867 & -1.711816 \\
$\mathrm{O}$ & -1.984239 & -1.066487 & 0.001977 \\
$\mathrm{O}$ & 0.975520 & -1.102623 & 1.713872 \\
$\mathrm{O}$ & -0.977916 & 1.096799 & -1.712260 \\
$\mathrm{O}$ & -0.976176 & 1.102696 & 1.713431 \\
$\mathrm{O}$ & 1.984230 & 1.066498 & 0.002707 \\
$\mathrm{C}$ & 1.558683 & -1.883068 & -2.705375 \\
$\mathrm{C}$ & 0.497631 & -2.794632 & -3.337814 \\
$\mathrm{C}$ & 2.665114 & -2.758733 & -2.099749 \\
$\mathrm{C}$ & 2.165020 & -0.985433 & -3.793733 \\
$\mathrm{C}$ & -3.129059 & -1.861025 & 0.000921 \\
$\mathrm{C}$ & -3.215072 & -2.655826 & 1.311822 \\
$\mathrm{C}$ & -4.378276 & -0.978979 & -0.136677 \\
$\mathrm{C}$ & -3.078546 & -2.848884 & -1.173167 \\
$\mathrm{C}$ & 1.493180 & -1.936951 & 2.702947 \\
$\mathrm{C}$ & 2.070593 & -3.206879 & 2.063104 \\
$\mathrm{C}$ & 0.382177 & -2.335563 & 3.686232 \\
$\mathrm{C}$ & 2.602998 & -1.209666 & 3.475166 \\
$\mathrm{C}$ & -1.557638 & 1.883056 & -2.706004 \\
$\mathrm{C}$ & -0.496325 & 2.794623 & -3.338003 \\
$\mathrm{C}$ & -2.664278 & 2.758721 & -2.100761 \\
$\mathrm{C}$ & -2.163583 & 0.985480 & -3.794627 \\
$\mathrm{C}$ & -1.494234 & 1.936977 & 2.702337 \\
$\mathrm{C}$ & -0.383612 & 2.335602 & 3.686047 \\
$\mathrm{C}$ & -2.604315 & 1.209633 & 3.474124 \\
$\mathrm{C}$ & -2.071454 & 3.206903 & 2.062315 \\
$\mathrm{C}$ & 3.129068 & 1.861010 & 0.002131 \\
$\mathrm{C}$ & 4.378319 & 0.978943 & -0.135023 \\
$\mathrm{C}$ & 3.214593 & 2.655747 & 1.313104 \\
$\mathrm{C}$ & 3.079031 & 2.848926 & -1.171928 \\
$\mathrm{H}$ & 0.912633 & -3.428564 & -4.116371 \\
$\mathrm{H}$ & -0.295512 & -2.201875 & -3.789608 \\
$\mathrm{H}$ & 0.054650 & -3.437373 & -2.582746 \\
$\mathrm{H}$ & 3.141526 & -3.391264 & -2.843487 \\
$\mathrm{H}$ & 2.255653 & -3.402040 & -1.326189 \\
& & & \\
\hline
\end{tabular}




$\begin{array}{lrrr}\mathrm{H} & 3.442004 & -2.139484 & -1.654929 \\ \mathrm{H} & 2.630289 & -1.561137 & -4.589149 \\ \mathrm{H} & 2.923721 & -0.334056 & -3.367649 \\ \mathrm{H} & 1.395619 & -0.361650 & -4.241015 \\ \mathrm{H} & -4.098173 & -3.287161 & 1.352529 \\ \mathrm{H} & -3.256523 & -1.981678 & 2.164960 \\ \mathrm{H} & -2.343849 & -3.296335 & 1.421536 \\ \mathrm{H} & -4.436747 & -0.273874 & 0.688352 \\ \mathrm{H} & -5.294591 & -1.562874 & -0.139889 \\ \mathrm{H} & -4.340691 & -0.412893 & -1.063240 \\ \mathrm{H} & -3.951100 & -3.495420 & -1.203263 \\ \mathrm{H} & -2.196390 & -3.477818 & -1.098153 \\ \mathrm{H} & -3.037469 & -2.315684 & -2.121358 \\ \mathrm{H} & 2.452327 & -3.902272 & 2.805571 \\ \mathrm{H} & 2.892811 & -2.959705 & 1.394764 \\ \mathrm{H} & 1.307114 & -3.714663 & 1.481490 \\ \mathrm{H} & -0.055383 & -1.451322 & 4.143129 \\ \mathrm{H} & 0.750764 & -2.971753 & 4.485765 \\ \mathrm{H} & -0.405455 & -2.882395 & 3.172677 \\ \mathrm{H} & 3.038602 & -1.834561 & 4.249969 \\ \mathrm{H} & 2.211012 & -0.314845 & 3.952125 \\ \mathrm{H} & 3.399331 & -0.908474 & 2.801059 \\ \mathrm{H} & -0.911017 & 3.428596 & -4.116691 \\ \mathrm{H} & 0.296976 & 2.201866 & -3.789519 \\ \mathrm{H} & -0.053617 & 3.437324 & -2.582741 \\ \mathrm{H} & -3.140397 & 3.391290 & -2.844653 \\ \mathrm{H} & -2.255095 & 3.401992 & -1.327024 \\ \mathrm{H} & -3.441349 & 2.139471 & -1.656257 \\ \mathrm{H} & -2.628536 & 1.561226 & -4.590198 \\ \mathrm{H} & -2.922461 & 0.334104 & -3.368855 \\ \mathrm{H} & -1.394028 & 0.361694 & -4.241640 \\ \mathrm{H} & -0.752520 & 2.971754 & 4.485462 \\ \mathrm{H} & 0.404190 & 2.882478 & 3.172800 \\ \mathrm{H} & 0.053813 & 1.451364 & 4.143082 \\ \mathrm{H} & -3.040231 & 1.834492 & 4.248779 \\ \mathrm{H} & -2.212475 & 0.314814 & 3.951209 \\ \mathrm{H} & -3.400385 & 0.908428 & 2.799711 \\ \mathrm{H} & -1.307775 & 3.714730 & 1.481002 \\ \mathrm{H} & -2.453491 & 3.902261 & 2.804659 \\ \mathrm{H} & -2.893412 & 2.959717 & 1.393659 \\ \mathrm{H} & 4.294648 & 1.562818 & -0.137855 \\ \mathrm{H} & & 0.412900 & -1.061626 \\ \mathrm{H} & -346458 & 0.273800 & 0.689997 \\ \mathrm{H} & 3.296277 & 1.422508 \\ \mathrm{H} & 3.287695 & 1.981560 & 2.166227\end{array}$




$\begin{array}{llll}\mathrm{H} & 3.951611 & 3.495443 & -1.201657 \\ \mathrm{H} & 2.196860 & 3.477875 & -1.097224 \\ \mathrm{H} & 3.038304 & 2.315771 & -2.120160\end{array}$


Octamer, $\left({ }^{\mathrm{t}} \mathrm{BuOLi}\right)_{8}, \mathbf{I V}$

HF/6-31G ${ }^{*}$ optimization: -1913.00602114 h Single Point B3LYP/6-311G**: $\mathrm{h}$

$\begin{array}{lccc}\mathrm{C} & -0.890020 & 4.034372 & -1.546309 \\ \mathrm{C} & -0.882798 & 4.070839 & 0.969475 \\ \mathrm{C} & 2.928279 & 4.070895 & -0.580551 \\ \mathrm{C} & -2.956072 & 3.493902 & -0.278104 \\ \mathrm{C} & -1.426081 & 3.358935 & -0.276030 \\ \mathrm{C} & 3.491331 & 3.188511 & 1.685762 \\ \mathrm{C} & 3.361360 & 2.822159 & 0.198425 \\ \mathrm{C} & 4.740824 & 2.392431 & -0.325601 \\ \mathrm{O} & -1.047296 & 2.008285 & -0.259579 \\ \mathrm{C} & 0.691130 & 1.115789 & -4.070422 \\ \mathrm{Li} & 0.735324 & 1.645375 & -0.888836 \\ \mathrm{C} & -3.719087 & 0.450322 & -3.34154 \\ \mathrm{O} & 2.429787 & 1.791450 & 0.046649 \\ \mathrm{Li} & -2.444435 & 0.809079 & -0.763976 \\ \mathrm{C} & -5.238427 & 0.457400 & 0.809157 \\ \mathrm{C} & -0.366074 & 1.431579 & 4.156445 \\ \mathrm{Li} & -1.184697 & 0.883373 & 1.361681 \\ \mathrm{C} & -4.032616 & 0.610463 & 2.974833 \\ \mathrm{C} & 3.026081 & 0.588200 & -3.394095 \\ \mathrm{C} & 1.567269 & 0.115961 & -3.304934 \\ \mathrm{O} & -2.867370 & 0.009961 & 0.945208 \\ \mathrm{Li} & 2.092125 & 0.654822 & 1.551703 \\ \mathrm{C} & -4.077888 & -0.126787 & 1.630171 \\ \mathrm{C} & -3.147253 & -0.914886 & -2.925830 \\ \mathrm{O} & 1.151305 & 0.040184 & -1.967310 \\ \mathrm{O} & 0.376426 & 0.180074 & 2.241273 \\ \mathrm{C} & 0.315690 & 0.155512 & 3.642599 \\ \mathrm{Li} & 2.589595 & 0.020157 & -0.712146 \\ \mathrm{O} & -2.254333 & -0.748373 & -1.863665 \\ \mathrm{C} & -2.446622 & -1.544595 & -4.136733 \\ \mathrm{C} & 1.726751 & 0.097032 & 4.246404 \\ \mathrm{C} & -4.310759 & -1.834138 & -2.521580 \\ \mathrm{C} & 1.478540 & -1.271191 & -3.956950 \\ \mathrm{Li} & -0.359835 & -1.106053 & -1.638656 \\ \mathrm{Li} & -2.237274 & -1.484056 & -0.075578 \\ \mathrm{C} & -4.359824 & -1.615085 & 1.892391 \\ \mathrm{C} & -0.459280 & -1.082362 & 4.111999 \\ \mathrm{O} & 2.691913 & -1.053038 & 0.871808 \\ \mathrm{C} & 4.808325 & -1.015699 & 2.038017\end{array}$




\begin{tabular}{|c|c|c|c|}
\hline $\mathrm{Li}$ & 0.809205 & -1.422695 & 1.165811 \\
\hline $\mathrm{C}$ & 4.587600 & -2.023745 & -0.236996 \\
\hline $\mathrm{O}$ & -0.480429 & -2.228542 & -0.014383 \\
\hline $\mathrm{C}$ & 3.863759 & -1.780490 & 1.097230 \\
\hline $\mathrm{C}$ & -0.456853 & -3.630404 & -0.061630 \\
\hline $\mathrm{C}$ & 3.550860 & -3.136776 & 1.742431 \\
\hline $\mathrm{C}$ & -1.796728 & -4.179129 & -0.574200 \\
\hline $\mathrm{C}$ & 0.650992 & -4.104259 & -1.011044 \\
\hline $\mathrm{C}$ & -0.222414 & -4.194755 & 1.346820 \\
\hline $\mathrm{H}$ & -1.175029 & 5.081407 & -1.599331 \\
\hline $\mathrm{H}$ & 0.193480 & 3.994168 & -1.588644 \\
\hline $\mathrm{H}$ & -1.278653 & 3.534515 & -2.428410 \\
\hline $\mathrm{H}$ & -1.093743 & 5.136735 & 0.953035 \\
\hline $\mathrm{H}$ & 0.188382 & 3.934760 & 1.048489 \\
\hline $\mathrm{H}$ & -1.334963 & 3.660505 & 1.868547 \\
\hline $\mathrm{H}$ & 3.676957 & 4.856189 & -0.522883 \\
\hline $\mathrm{H}$ & 2.000526 & 4.471810 & -0.191288 \\
\hline $\mathrm{H}$ & 2.782411 & 3.830376 & -1.630085 \\
\hline $\mathrm{H}$ & -3.264598 & 4.535217 & -0.291719 \\
\hline $\mathrm{H}$ & -3.392021 & 3.024157 & -1.158785 \\
\hline $\mathrm{H}$ & -3.383027 & 3.032762 & 0.604524 \\
\hline $\mathrm{H}$ & 4.171814 & 4.020158 & 1.845080 \\
\hline $\mathrm{H}$ & 2.523493 & 3.464978 & 2.094120 \\
\hline $\mathrm{H}$ & 3.877197 & 2.346967 & 2.258862 \\
\hline $\mathrm{H}$ & 5.486074 & 3.167709 & -0.170598 \\
\hline $\mathrm{H}$ & 4.701058 & 2.182966 & -1.389088 \\
\hline $\mathrm{H}$ & 5.086044 & 1.498256 & 0.179553 \\
\hline $\mathrm{H}$ & 0.825599 & 2.118920 & -3.674438 \\
\hline $\mathrm{H}$ & 0.936909 & 1.144878 & -5.128556 \\
\hline $\mathrm{H}$ & -0.355898 & 0.859676 & -3.968812 \\
\hline $\mathrm{H}$ & -2.916365 & 1.134424 & -3.601044 \\
\hline $\mathrm{H}$ & -4.383445 & 0.373158 & -4.197162 \\
\hline $\mathrm{H}$ & -4.295468 & 0.891138 & -2.529366 \\
\hline $\mathrm{H}$ & -5.103699 & 1.522550 & 0.655002 \\
\hline $\mathrm{H}$ & -6.192474 & 0.315411 & 1.309271 \\
\hline $\mathrm{H}$ & -5.302611 & -0.016593 & -0.163015 \\
\hline $\mathrm{H}$ & 0.185116 & 2.308041 & 3.829336 \\
\hline $\mathrm{H}$ & -1.381298 & 1.515675 & 3.782774 \\
\hline $\mathrm{H}$ & -0.420166 & 1.453087 & 5.241310 \\
\hline $\mathrm{H}$ & -3.787836 & 1.658269 & 2.823728 \\
\hline $\mathrm{H}$ & -4.990145 & 0.565673 & 3.486364 \\
\hline $\mathrm{H}$ & -3.288090 & 0.178552 & 3.632188 \\
\hline $\mathrm{H}$ & 3.139796 & 1.568840 & -2.947569 \\
\hline $\mathrm{H}$ & 3.362723 & 0.650907 & -4.424848 \\
\hline $\mathrm{H}$ & 3.696061 & -0.103300 & -2.884914 \\
\hline $\mathrm{H}$ & -1.671338 & -0.893569 & -4.521545 \\
\hline
\end{tabular}




$\begin{array}{lrrr}\mathrm{H} & -3.145887 & -1.738670 & -4.945371 \\ \mathrm{H} & -1.990988 & -2.491421 & -3.860025 \\ \mathrm{H} & 2.313425 & 0.969708 & 3.962575 \\ \mathrm{H} & 1.695617 & 0.078370 & 5.331942 \\ \mathrm{H} & 2.249672 & -0.791823 & 3.914042 \\ \mathrm{H} & -5.037399 & -1.934458 & -3.323085 \\ \mathrm{H} & -4.829431 & -1.444177 & -1.653929 \\ \mathrm{H} & -3.950399 & -2.827909 & -2.278642 \\ \mathrm{H} & 1.805304 & -1.253387 & -4.992918 \\ \mathrm{H} & 0.461160 & -1.648333 & -3.947780 \\ \mathrm{H} & 2.102897 & -1.978171 & -3.419034 \\ \mathrm{H} & -5.270076 & -1.764938 & 2.466029 \\ \mathrm{H} & -4.481014 & -2.156472 & 0.955510 \\ \mathrm{H} & -3.539010 & -2.065116 & 2.443425 \\ \mathrm{H} & -1.436541 & -1.118097 & 3.647380 \\ \mathrm{H} & -0.590672 & -1.094960 & 5.190646 \\ \mathrm{H} & 0.071250 & -1.989618 & 3.835267 \\ \mathrm{H} & 5.046026 & -0.037482 & 1.637396 \\ \mathrm{H} & 4.352953 & -0.877620 & 3.012713 \\ \mathrm{H} & 5.743736 & -1.548671 & 2.184401 \\ \mathrm{H} & 4.899416 & -1.081613 & -0.684983 \\ \mathrm{H} & 3.931896 & -2.534284 & -0.936498 \\ \mathrm{H} & 5.481663 & -2.628384 & -0.113956 \\ \mathrm{H} & 2.996304 & -2.997240 & 2.666361 \\ \mathrm{H} & 2.958786 & -3.756810 & 1.080623 \\ \mathrm{H} & 4.458942 & -3.683210 & 1.981865 \\ \mathrm{H} & -2.006389 & -3.809771 & -1.570989 \\ \mathrm{H} & -2.617452 & -3.890563 & 0.081127 \\ \mathrm{H} & -1.793703 & -5.264489 & -0.615368 \\ \mathrm{H} & 0.438171 & -3.789798 & -2.029365 \\ \mathrm{H} & 1.604099 & -3.676333 & -0.727049 \\ \mathrm{H} & 0.747554 & -5.186646 & -1.015115 \\ \mathrm{H} & -1.009311 & -3.864378 & 2.018118 \\ \mathrm{H} & 0.726695 & -3.861502 & 1.753656 \\ \mathrm{H} & -0.210093 & -5.281104 & 1.350942\end{array}$




\section{Cryoscopic Determinations.}

The cryoscopic apparatus comprised an inner sample tube surrounded by an air jacket, which was cooled by an ethanol bath $\left(\mathrm{ca} .-20{ }^{\circ} \mathrm{C}\right) .{ }^{1}$ The temperature of the latter was controlled by a cryostat. The sample was agitated using a manual stirrer to ensure complete mixing. The freezing point depressions were measured using a Philadelphia Differential Thermometer (reading to ca. $0.001{ }^{\circ} \mathrm{C}$ ). Anhydrous Benzene (supplied by Aldrich) was used for the molecular weight determinations. The benzene cryoscopic constant $\mathrm{k}_{\mathrm{b}}$ was determined using freshly sublimed biphenyl $(\mathrm{MW}=154.211)$. The freezing point of benzene was determined before a known mass of a complex was introduced into the apparatus. The new freezing point was then determined and from equation 1 the molecular mass MW was calculated. The depression in freezing point was measured three times at each concentration.

$\mathrm{MW}=1000 \cdot \mathrm{g} \cdot \mathrm{k}_{\mathrm{b}} \cdot(\mathrm{G} \cdot \Delta \mathrm{T})^{-1} \quad($ eq. 1$)$

MW: Molecular weight of the substrate.

g: Weight of the solute..

G: Weight of the solvent.

$\Delta \mathrm{T}$ : Freezing point depression.

The results obtained for the octamer $\mathbf{1}_{8}$ were as follows:

$5.1 \mathrm{mmol} \mathrm{L}^{-1}$ : 646, $11.1 \mathrm{mmol} \mathrm{L}^{-1}: 633,14.2 \mathrm{mmol} \mathrm{L}^{-1}: 643,16.7 \mathrm{mmol} \mathrm{L}{ }^{-1}: 666$.

The results obtained for the hexamer $\mathbf{1}_{6}$ were as follows:

$10.7 \mathrm{mmol} \mathrm{L}^{-1}$ : 453, $11.2 \mathrm{mmol} \mathrm{L}^{-1}$ : 490, $19.0 \mathrm{mmol} \mathrm{L}^{-1}$ : 494, $21.1 \mathrm{mmol} \mathrm{L}{ }^{-1}: 449$.

A sample of the octamer in benzene was heated to reflux temperature and its molecular weight determined after various time intervals as shown below.

0 min: 643, 55 min: 573, $105 \min : 447,145 \min : 445$.

[1] Experiments in Physical Chemistry; $5^{\text {th }}$ Edn.; Shoemaker, D. P.; Garland, C. W.; Nibler, J. W.; McGraw-Hill: New York, 1989. 


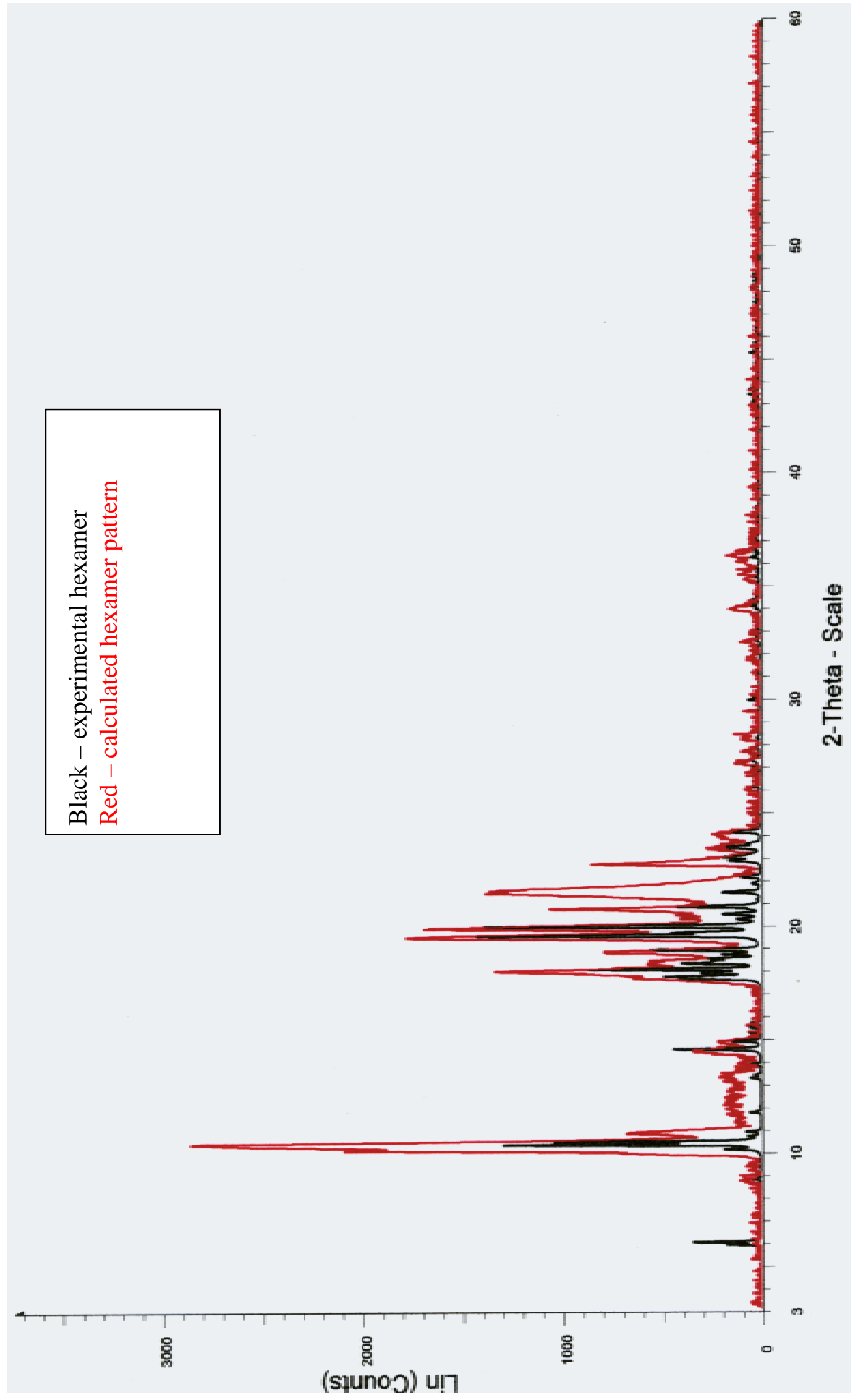




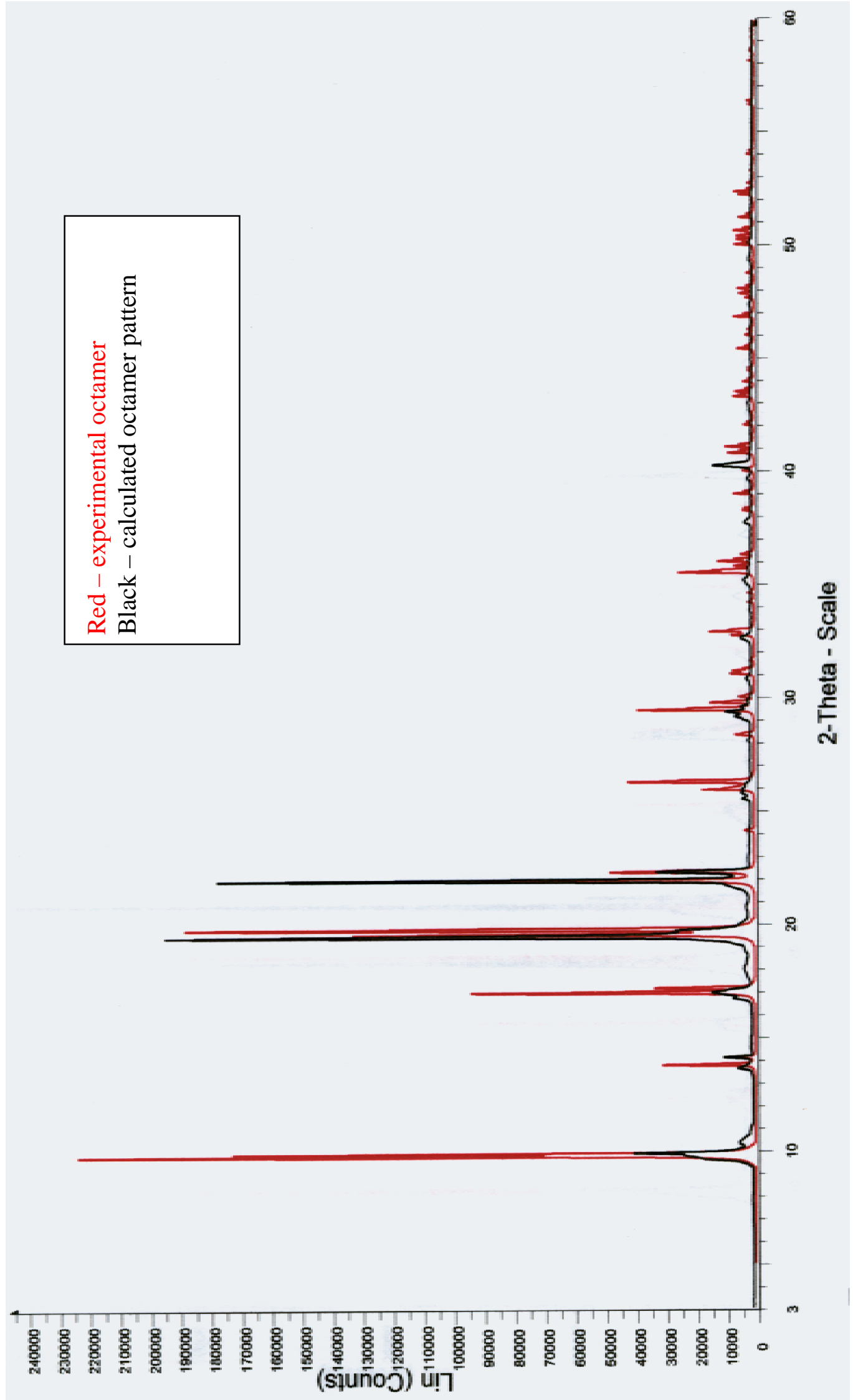




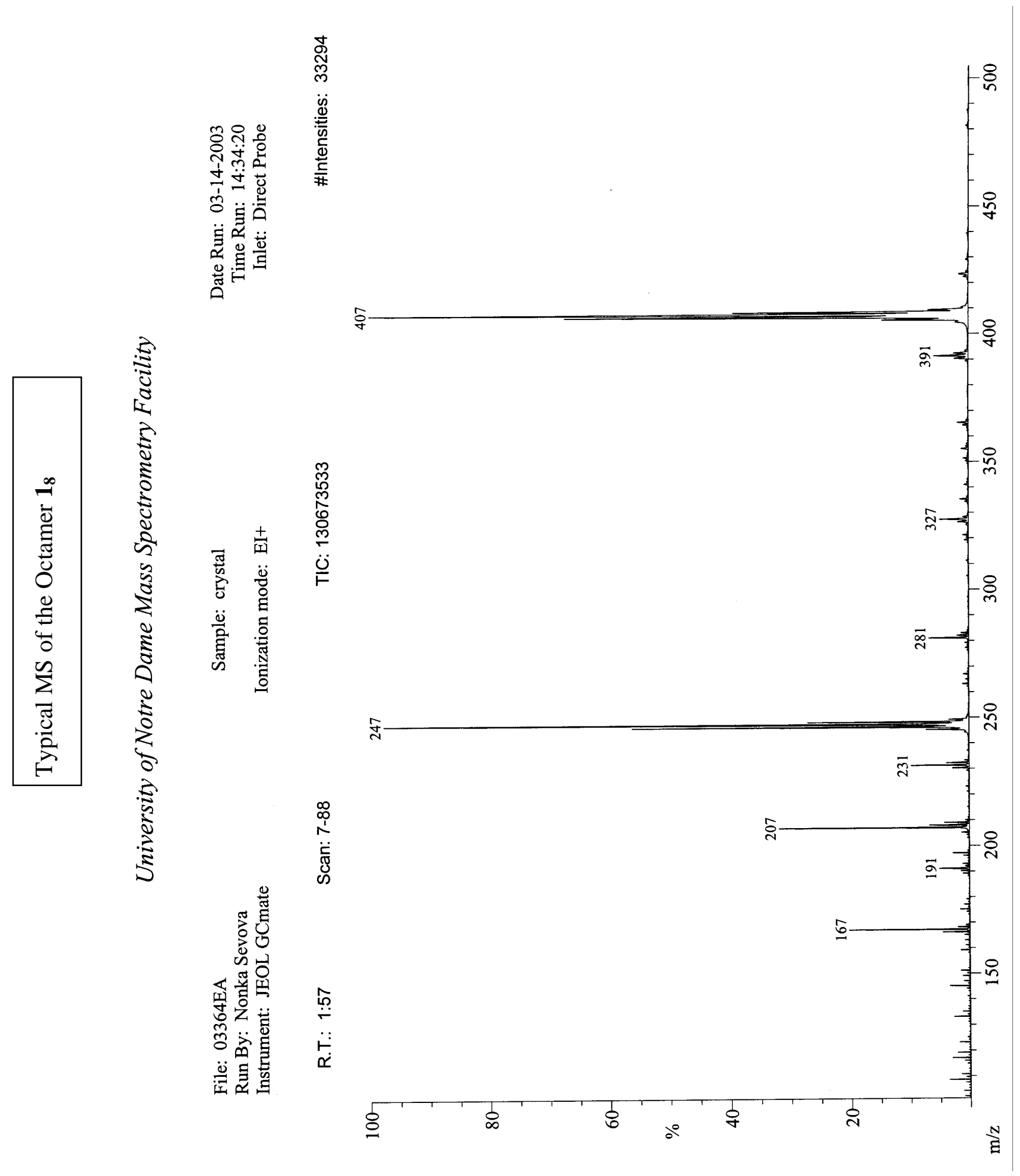




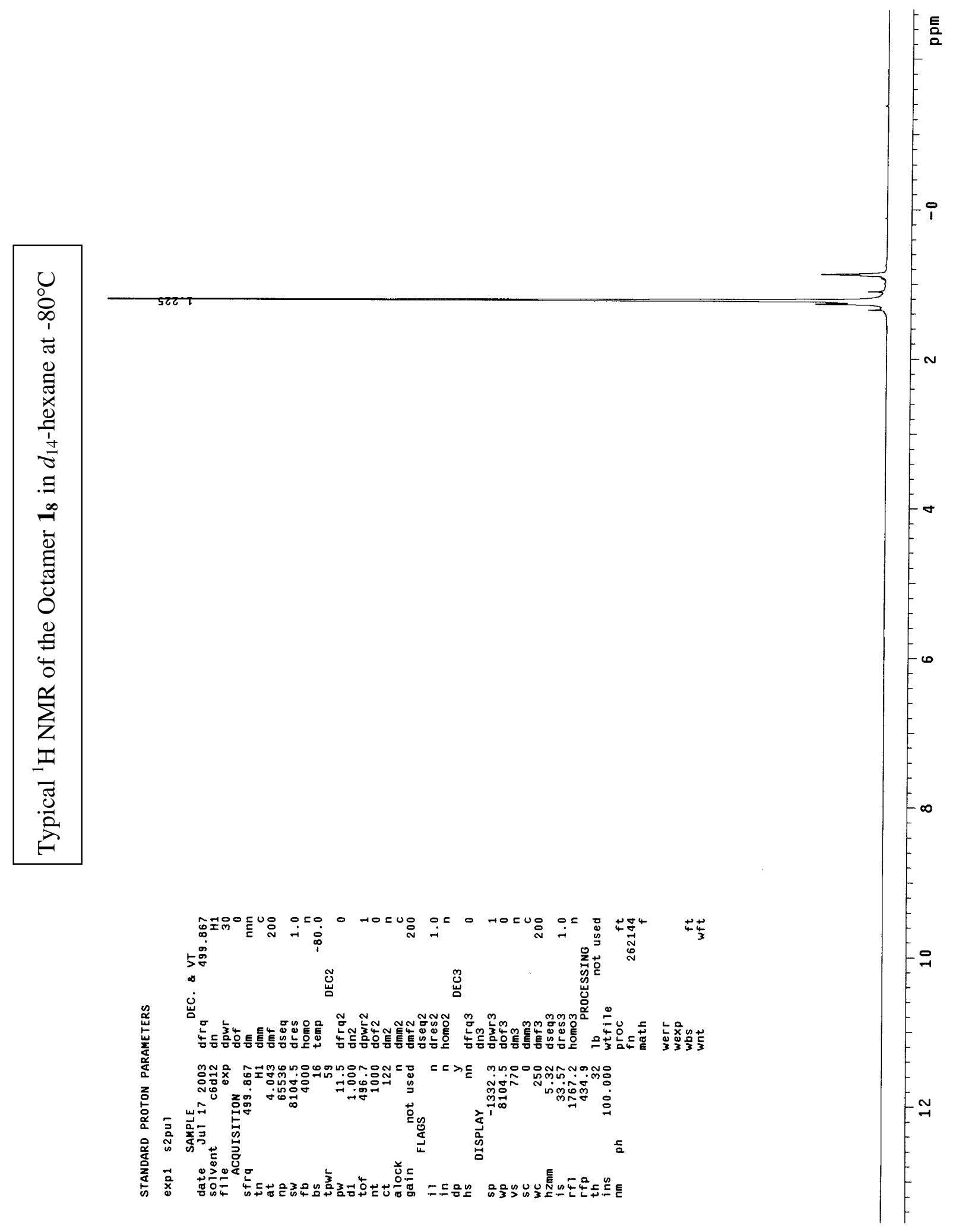




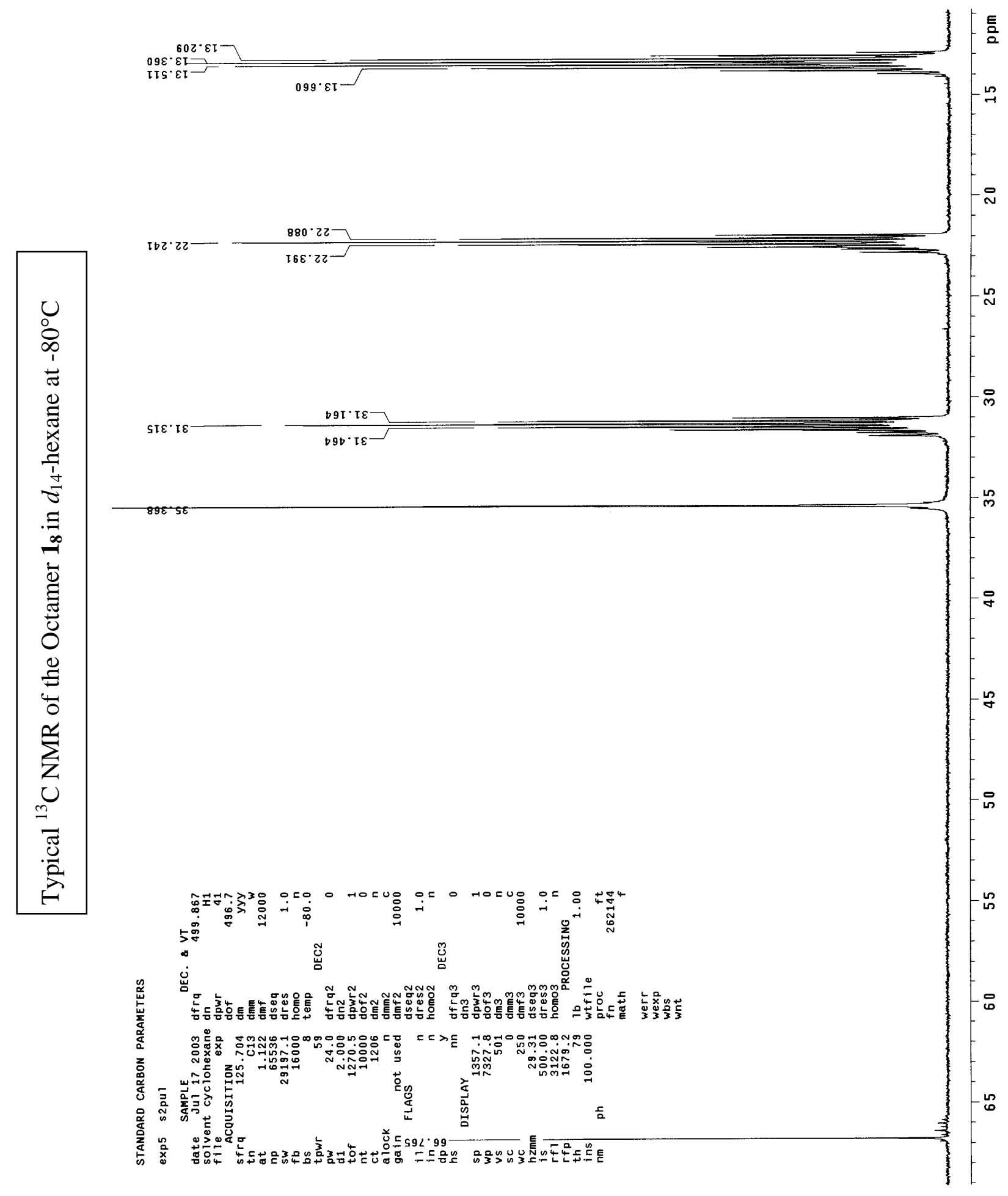




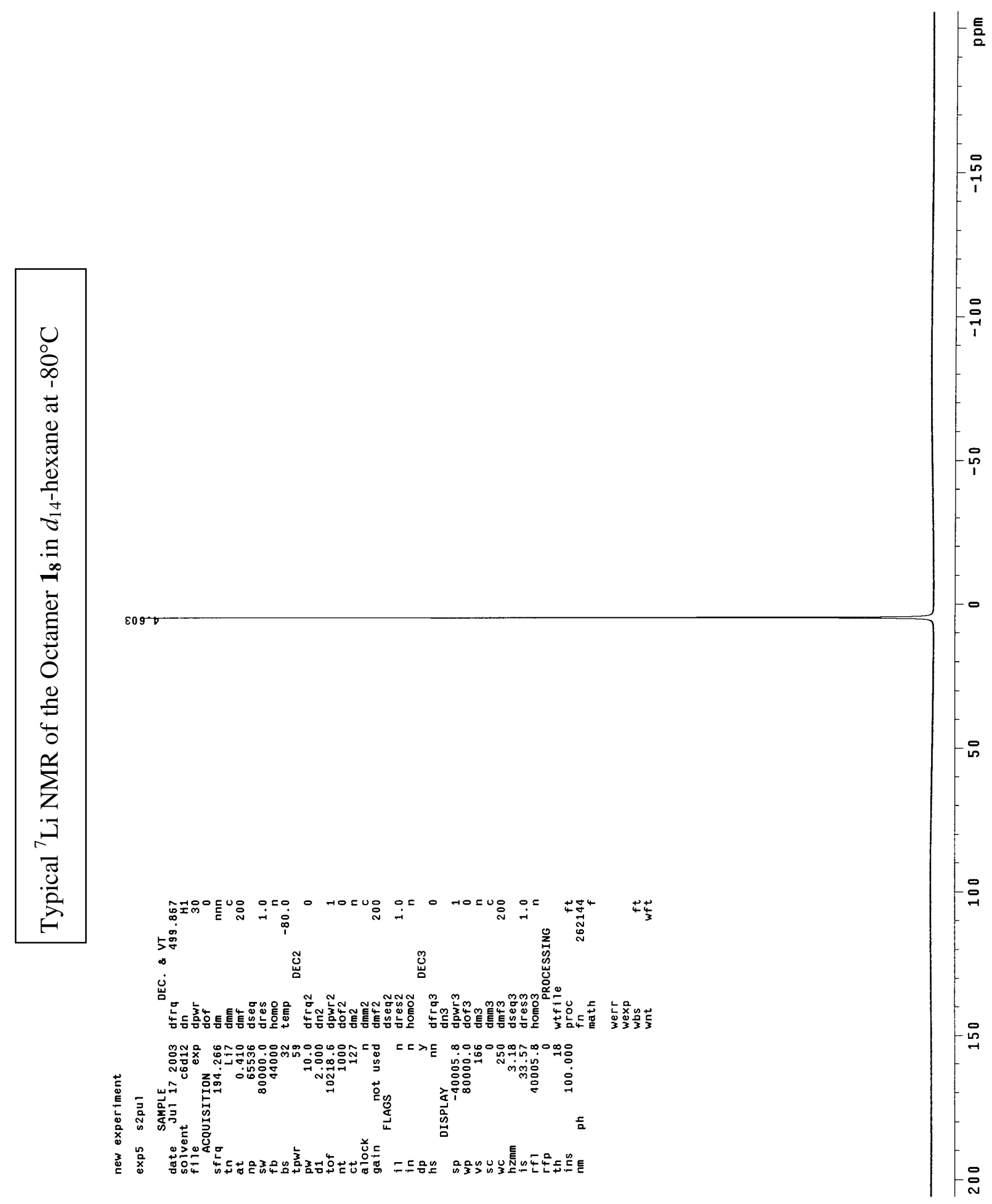

